\title{
On the generalized continued fractions
}

\author{
Amara Chandoul ${ }^{1}$ and Fahad Aljuaydi ${ }^{2}$ \\ ${ }^{1}$ Higher Institute of Informatics and Multimedia of Sfax, Sfax University, Tunisia. \\ ${ }^{2}$ Department of mathematics, College of Sciences and Humanities, Prince Sattam bin Abdulaziz University, Al-Kharj, Saudi Arabia.
}

Received: 20 January 2019, Accepted: 4 March 2019

Published online: 4 October 2019.

\begin{abstract}
We introduce a class of continued fractions called Oppenheim continued fractions (OCF). Basic properties of these expansions are discussed and studied in the formal powers series case.
\end{abstract}

Keywords: Oppenhein continued fraction, Laurent series, finite fields.

\section{On generalized continued fractions}

Let $\mathbb{F}_{q}$ be a finite field with $q$ elements of characteristic $p, \mathbb{F}_{q}[X]$ the set of polynomials of coefficients in $\mathbb{F}_{q}$ and $\mathbb{F}_{q}(X)$ its field of fractions. The set $\mathbb{F}_{q}\left(\left(X^{-1}\right)\right)$ is the field of formal power series over $\mathbb{F}_{q}$

$$
\mathbb{F}_{q}\left(\left(X^{-1}\right)\right)=\left\{\omega=\sum_{j=s}^{+\infty} a_{j} X^{-j}: a_{j} \in \mathbb{F}_{q}, s \in \mathbb{Z}\right\}
$$

Let $\omega=\sum_{j=s}^{+\infty} a_{j} X^{-j} \in \mathbb{F}_{q}\left(\left(X^{-1}\right)\right)$, where $a_{s} \neq 0$. We denote its polynomial part by $[\omega]$ and $\{\omega\}$ its fractional part. We remark that $\omega=[\omega]+\{\omega\}$. We define a non-archimedean absolute value on $\mathbb{F}_{q}\left(\left(X^{-1}\right)\right)$ by $|\omega|=e^{-s}$. It is clear that, for all $P \in \mathbb{F}_{q}[X],|P|=e^{\operatorname{deg} P}$ and, for all $Q \in \mathbb{F}_{q}[X]$, such that $Q \neq 0,\left|\frac{P}{Q}\right|=e^{\operatorname{deg} P-\operatorname{deg} Q}$.

Let $E=\left(\mathbb{F}_{q}\left(\left(X^{-1}\right)\right)\right)^{n}, E$ is a vectorial space over $\mathbb{F}_{q}\left(\left(X^{-1}\right)\right)$. We define a norm over $E$ as follows, for all $f=\left(f_{1}, \ldots, f_{n}\right) \in E$,

$$
\|f\|=\max _{1 \leq i \leq n}\left|f_{i}\right|
$$

Let $A_{1}, \ldots, A_{m} \in E$, then we can verify that

$$
\left\|A_{1}+\cdots+A_{m}\right\| \leq \max _{1 \leq i \leq m}\left\|A_{i}\right\|
$$

We begin by giving a few basics facts about the generalized continued fractions over $\mathbb{F}_{q}\left(\left(X^{-1}\right)\right)$. 


\subsection{Basics concepts}

Let $\left(\alpha_{n}\right)_{n \in \mathbb{N}}$ and $\left(\beta_{n}\right)_{n \in \mathbb{N}} \in \mathbb{F}_{q}\left(\left(X^{-1}\right)\right)$, a continued fraction

$$
K\left(\frac{\alpha_{n}}{\beta_{n}}\right)=\frac{\alpha_{1}}{\beta_{1}+\frac{\alpha_{2}}{\beta_{2}+\frac{\alpha_{3}}{\beta_{3}+\ddots}}}
$$

is said to converge if its sequence of approximants $\left\{\omega_{n}\right\}$ converges. Here

$$
\omega_{n}=K_{i=1}^{n}\left(\frac{\alpha_{i}}{\beta_{i}}\right)=\frac{\alpha_{1}}{\beta_{1}+\frac{\alpha_{2}}{\beta_{2}+\frac{\alpha_{3}}{\ddots+\frac{\alpha_{n}}{\beta_{n}}}}} \text {, for } n=1,2, \ldots
$$

The value of the continued fraction is then $\omega=K\left(\frac{\alpha_{n}}{\beta_{n}}\right)=\lim _{n \rightarrow+\infty} \omega_{n}$.

Remark. If $\alpha_{i}=1$ and $\beta_{i}$ is a non constant polynomial, then we obtain the Regular continued fraction (RCF).

If $\alpha_{i}$ is a fixed polynomial $P$ and $\left.\left(\beta_{i}\right)_{i \geq 1}\right)$ is a sequence of non constant polynomials, then we obtain the $P$-continued fraction.

If $K\left(\frac{\alpha_{n}}{\beta_{n}}\right)$ converges, its tails $K_{n=N+1}^{+\infty}\left(\frac{\alpha_{n}}{\beta_{n}}\right)$ for $N=0,1,2, \ldots$ also converge, and we let $\omega^{(N)}=K_{n=N+1}^{+\infty}\left(\frac{\alpha_{n}}{\beta_{n}}\right)$ denote the values of these tails for $N=0,1,2, \ldots$. It is easy to see that $\left\{\omega^{(N)}\right\}$ is a sequence with $\omega^{(0)}=\omega$, satisfying the recursion relations

$$
\omega^{(N)}=\frac{\alpha_{N+1}}{\beta_{N+1}+\omega^{(N+1)}} \text { for } N=1,2, \ldots
$$

This sequence is what Waadeland [10] named the sequence of right tails for $K\left(\frac{\alpha_{n}}{\beta_{n}}\right)$.

In this section, we describe a necessary and sufficient conditions for the convergence of (1). For which, we assume the existence of the limits

$$
\lim _{n \rightarrow+\infty} \alpha_{n}=\alpha \neq 0 \text { and } \lim _{n \rightarrow+\infty} \beta_{n}=\beta .
$$

The continued fraction expansion (1) can be generated by means of the sequence $\left\{s_{n}(\theta)\right\}$ of linear fractional transformations,

$$
s_{n}(\theta)=\frac{\alpha_{n}}{\beta_{n}+\theta}, \text { for } \theta \in \mathbb{F}_{q}\left(\left(X^{-1}\right)\right) \text { and } n=1,2,3, \ldots
$$

Defining $S_{n}(\theta)$ as their composition,

$$
S_{0}(\theta)=\theta, S_{n}(\theta)=S_{n-1}\left(s_{n}(\theta)\right) \text { for and } n=1,2,3, \ldots
$$

gives us $\omega_{n}=S_{n}(0)$, from (2). Straightforward computation shows that $S_{n}(\theta)$ can be written

$$
S_{n}(\theta)=\frac{A_{n}+A_{n-1} \theta}{B_{n}+B_{n-1} \theta} \text { for } n=0,1,2, \ldots
$$

where $A_{n}$ and $B_{n}$, the numerator and denominator of $K_{i=0}^{n}\left(\frac{\alpha_{i}}{\beta_{i}}\right)$, respectively, are given by

$$
A_{-1}=1, A_{0}=0, A_{n}=\beta_{n} A_{n-1}+\alpha_{n} A_{n-2}, \text { for } n=1,2, \ldots
$$




$$
B_{-1}=1, B_{0}=0, B_{n}=\beta_{n} B_{n-1}+\alpha_{n} B_{n-2}, \text { for } n=1,2, \ldots
$$

This notation is in accordance with [9], and it will be used throughout this paper. If we regard the $N$ th tail $K_{m=N+1}^{+\infty}\left(\frac{\alpha_{m}}{\beta_{m}}\right)$ as a continued fraction, we use the notation $S_{n}^{(N)}, A_{n}^{(N)}$ and $B_{n}^{(N)}$ to denote the similar expressions connected with $K_{m=N+1}^{+\infty}\left(\frac{\alpha_{m}}{\beta_{m}}\right)$.

\subsection{Convergence results}

Theorem 1. Let $\left(\alpha_{n}\right)_{n \in \mathbb{N}}$ and $\left(\beta_{n}\right)_{n \in \mathbb{N}} \in \mathbb{F}_{q}\left(\left(X^{-1}\right)\right)[z]$. If in the generalized continued fraction

$$
\omega(z)=K_{n=1}^{+\infty} \frac{\alpha_{n}(z)}{\beta_{n}(z)}
$$

$\lim _{i \rightarrow+\infty} \alpha_{i}(z)=\alpha(z) \neq 0$ and $\lim _{i \rightarrow+\infty} \beta_{i}(z)=\beta(z)$, the continued fraction expansion (10) will converge if and only if $z \in\left\{z \in \mathbb{F}_{q}\left(\left(X^{-1}\right)\right) ; a(z)=\frac{|\alpha(z)|}{|\beta(z)|^{2}}<1\right\}$ except possibly at certain isolated points $p_{1}, p_{1}, \ldots$, which are poles.

Proof. If a sufficient number of terms of (10) are omitted at the outset in which $\left(\left|\alpha_{N+i}(z)\right|,\left|\beta_{N+i}(z)\right|\right)=(|\alpha(z)|,|\beta(z)|)$ $\forall i \geq 1$, a new continued fraction will be obtained

$$
\omega^{(N)}(z)=K_{i=1}^{+\infty} \frac{\alpha_{N+i}(z)}{\beta_{N+i}(z)} .
$$

For this continued fraction

$$
B_{0}^{(N)}=1, B_{1}^{(N)}=\beta_{N+1}(z) \text { and } B_{i+1}^{(N)}=\beta_{N+i+1}(z) B_{i}^{(N)}+\alpha_{N+i+1}(z) B_{i-1}^{(N)}
$$

Suppose first that $|a(z)|=\frac{|\alpha(z)|}{|\beta(z)|^{2}}<1$, then, for all $i \geq 1$,

$$
\frac{\left|\beta_{N+i}(z)\right|^{2}}{\left|\alpha_{N+i}(z)\right|}=\frac{1}{|a(z)|}>1
$$

Let us proof that

$$
\left|B_{n}^{(N)}\right|=|\beta(z)|^{n} .
$$

If $\left|B_{s}^{(N)}\right|=|\beta(z)|^{s}$ for $s \leq n$, then $\left|\beta_{N+n+1}(z) B_{n}^{(N)}\right|=|\beta(z)|^{n+1}$ and $\left|\alpha_{N+n+1}(z) B_{n-1}^{(N)}\right|=|\alpha(z)||\beta(z)|^{n-1}$. We have immediately from (12) and (13).

$$
\left|B_{n+1}^{(N)}\right|=\left|\beta_{N+n+1}(z) B_{n}^{(N)}\right|=|\beta(z)|^{n+1} .
$$

We claim that the sequence $\left(\frac{A_{n}^{(N)}}{B_{n}^{(N)}}\right)_{n}$ converges. The difference between the $(n-1)$ th and the $n$th $(n>0)$ convergent is

$$
\frac{A_{n}^{(N)}}{B_{n}^{(N)}}-\frac{A_{n-1}^{(N)}}{B_{n-1}^{(N)}}=\frac{(-1)^{n} \prod_{i=1}^{n} \alpha_{N+i}(z)}{B_{n-1}^{(N)} B_{n}^{(N)}}
$$


Then from (14), $\left|\frac{A_{n}^{(N)}}{B_{n}^{(N)}}-\frac{A_{n-1}^{(N)}}{B_{n-1}^{(N)}}\right|=\frac{|\alpha(z)|^{n}}{|\beta(z)|^{2 n-1}}$.

Consequently for $k \in \mathbb{N}$,

$$
\left|\frac{A_{n+k}^{(N)}}{B_{n+k}^{(N)}}-\frac{A_{n}^{(N)}}{B_{n}^{(N)}}\right|=\frac{|\alpha(z)|^{n}}{|\beta(z)|^{2 n-1}}=|a(z)|^{n}|\beta(z)| \longrightarrow 0 .
$$

Now, suppose that $|a(z)| \geq 1$, one shows, using a simple recurrence on $n$ and (12) that

$$
\left|B_{2 n}^{(N)}\right| \leq|\alpha(z)|^{n} \text { and }\left|B_{2 n+1}^{(N)}\right| \leq|\beta(z)||\alpha(z)|^{n}
$$

Now, we are able to prove the divergence of $\frac{A_{n}^{(N)}(z)}{B_{n}^{(N)}(z)}$ under the assumption $|a(z)| \geq 1$. Indeed, if $\frac{A_{n}^{(N)}(z)}{B_{n}^{(N)}(z)}$ converge, then from (15), we deduce that

$$
\omega^{(N)}(z)=\sum_{k=1}^{+\infty} \frac{(-1)^{k} \prod_{i=1}^{k} \alpha_{N+i}(z)}{B_{k}^{(N)} B_{k-1}^{(N)}}
$$

then $A_{N}^{(N)}(z)$ diverge since from (16)

$$
\left|\frac{(-1)^{k} \prod_{i=1}^{k} \alpha_{N+i}(z)}{B_{k}^{(N)} B_{k-1}^{(N)}}\right| \geq\left|\frac{\alpha}{\beta}\right|>0
$$

\section{Oppenheim continued fraction expansions (OCF)}

Now, we introduce Oppenheim continued fraction expansion. Let $\mathscr{J}=\left\{\omega \in \mathbb{F}_{q}\left(\left(X^{-1}\right)\right):|\omega|<1\right.$ and $\left.\omega \neq 0\right\}$ and $\left\{h_{j}\right\}_{j \geq 1}$ be a sequence of polynomials valued map defined on $\mathbb{F}_{q}[X]$. Let $\omega \in \mathscr{J}$, as in the real case [8] we define the Oppenheim algorithm $T_{0}$ by

$$
T_{0}(\omega)=\frac{1}{h_{1}\left(D_{1}\right)+1}\left(\frac{1}{\omega}-D_{1}\right) \in \mathscr{J} \text { where } D_{1}=\left[\frac{1}{\omega}\right] .
$$

Now we define the polynomials $D_{j}=D_{j}(\omega)$ and the formal power series $\omega_{j}$ for $j=1,2, \ldots$ as follows :

$$
\left\{\begin{array}{l}
\omega_{1}=\omega, \quad D_{j}=\left[\frac{1}{\omega_{j}}\right], \\
\omega_{j+1}=T_{0}^{j}(\omega)=T_{0}\left(T_{0}^{j-1}(\omega)\right)=\frac{1}{h_{j}\left(D_{j}\right)+1}\left(\frac{1}{\omega_{j}}-\left[\frac{1}{\omega_{j}}\right]\right)
\end{array}\right.
$$

This algorithm generates the Oppenheim continued fraction expansion of $\omega$ as follows

$$
\omega=\frac{1}{D_{1}+\frac{h_{1}\left(D_{1}\right)+1}{D_{2}+\frac{h_{2}\left(D_{2}\right)+1}{D_{3}+\ddots+\frac{h_{j-1}\left(D_{j-1}\right)+1}{D_{j}+\ddots}}}},
$$

where $D_{j} \in F_{q}[X] \backslash F_{q}$

Proposition 1. we have

$$
\left|D_{j+1}\right|>\left|h_{j}\left(D_{j}\right)+1\right| \text { for all } j \geq 1
$$


In fact,

$$
D_{j+1}=\left[\frac{1}{T_{0}^{j}(\omega)}\right]=\left[\frac{h_{j}\left(D_{j}\right)+1}{\left\{\frac{1}{T_{0}^{j-1}(\omega)}\right\}}\right]
$$

then $\left|D_{j+1}\right|>\left|h_{j}\left(D_{j}\right)+1\right|$.

Proposition 2.Let $A_{n}$ and $B_{n}$, the numerator and denominator of $K_{i=0}^{n}\left(\frac{h_{i}\left(D_{i}\right)+1}{D_{i}}\right)$, then from (8) and (9), $\left(A_{n}\right),\left(B_{n}\right)$ are recursively defined by

$$
\begin{array}{llll}
A_{0}=0, & A_{1}=1, \quad A_{n}=D_{n} A_{n-1}+\left(h_{n-1}\left(D_{n-1}\right)+1\right) A_{n-2}, & \text { for } & n \geq 2 \\
B_{0}=1, & B_{1}=B_{1}, \quad B_{n}=D_{n} B_{n-1}+\left(h_{n-1}\left(D_{n-1}\right)+1\right) B_{n-2}, \quad \text { for } & n \geq 2
\end{array}
$$

Then, for $n \geq 2$

$$
A_{n} B_{n-1}-A_{n-1} B_{n}=(-1)^{n} \prod_{j=1}^{n-1}\left(h_{n-1}\left(D_{n-1}\right)+1\right)
$$

and

$$
\begin{gathered}
\frac{1}{D_{1}+\frac{\left(h_{1}\left(D_{1}\right)+1\right)}{D_{2}+\frac{\left(h_{2}\left(D_{2}\right)+1\right)}{D_{3}+\cdot+\frac{\left(h_{n-1}\left(D_{n-1}\right)+1\right)}{D_{n}}}}} \\
\left|B_{n}\right|>\left|A_{n}\right| \\
\left|B_{n+1}\right| \geq\left|\prod_{i=0}^{n} h_{i}\left(D_{i}+1\right)\right|
\end{gathered}
$$

Remark. (i) It is clear that the Oppenheim continued fraction is a particular case of the generalized continued fraction (1).

(ii) If $h_{j}\left(D_{j}\right)=0$, then we obtain the Regular continued fraction (RCF).

(iii) If $h_{j}\left(D_{j}\right)=D_{j}-1$, then we obtain the Engel continued fraction (ECF).

Proposition 3. A formal power series $\omega \in \mathscr{J}$ has a finite Oppenheim continued fraction expansion if and only if $\omega \in$ $\mathbb{F}_{q}(X)$.

Proof. Using the expression (19) of $\omega$, we state that if $\omega$ has a finite expansion then $\omega \in \mathbb{F}_{q}(X)$. Suppose now $\omega$ is rational fraction. By the algorithm, we know that for $j \geq 1, \omega_{j}$ is a rational fraction in $\mathscr{J}$, then $\omega_{j}:=\frac{R_{j}}{S_{j}}=\frac{R_{j}}{D_{j} R_{j}+R_{j+1}}$ where $\left|R_{j+1}\right|<\left|R_{j}\right|$ and $D_{j}=\left[\frac{S_{j}}{R_{j}}\right]$. Thus, by the algorithm, we have

$$
\omega_{j+1}=\frac{1}{h_{j}\left(D_{j}\right)+1}\left(\frac{1}{\omega_{j}}-D_{j}\right)=\frac{1}{h_{j}\left(D_{j}\right)+1} \frac{R_{j+1}}{R_{j}}:=\frac{R_{j+1}}{S_{j+1}} .
$$

Since $\left|R_{j+1}\right|<\left|R_{j}\right|$, then this procedure will stop at finite steps, it follows that $\omega_{j}=0$ for some $j$. 
Proposition 4. For all $\omega \in \mathscr{J}$, we have

$$
\lim _{n \rightarrow+\infty} \frac{A_{n}(\omega)}{B_{n}(\omega)}=\omega
$$

Proof. If $\omega$ is rational we conclude (28) by (20). Now let $\omega$ be irrational, (24) implies that

$$
\begin{gathered}
\omega=\frac{A_{n}(\omega)+\left(h_{n}\left(D_{n}(\omega)\right)+1\right) \omega_{n+1} A_{n-1}(\omega)}{B_{n}(\omega)+\left(h_{n}\left(D_{n}(\omega)\right)+1\right) \omega_{n+1} B_{n-1}(\omega)} \\
\left|\omega-\frac{A_{n}}{B_{n}}\right|=\frac{\left|\left(h_{n}\left(D_{n}(\omega)\right)+1\right) \omega_{n+1} \prod_{j=1}^{n-1}\left(h_{j}\left(D_{j}\right)+1\right)\right|}{\left|B_{n}\right|\left|\left(B_{n}+\left(h_{n}\left(D_{n}(\omega)\right)+1\right) \omega_{n+1} B_{n-1}\right)\right|} .
\end{gathered}
$$

Since $\left|\left(h_{n}\left(D_{n}(\omega)\right)+1\right) \omega_{n+1}\right|<1,\left|B_{n-1}\right|<\left|B_{n}\right|$ and $\left|\prod_{j=1}^{n-1}\left(h_{j}\left(D_{j}\right)+1\right)\right|<\left|B_{n}\right|$, then

$$
\left|\omega-\frac{A_{n}}{B_{n}}\right|<\frac{1}{\left|B_{n}\right|} \longrightarrow 0
$$

Proposition 5. Let $\left(D_{1}, \ldots, D_{n}, \ldots\right)$ and $\left(h_{1}\left(D_{1}\right), \ldots, h_{n}\left(D_{n}\right), \ldots\right)$ be two sequences of polynomials such that $\left|D_{i+1}\right|>\mid$ $h_{i}\left(D_{i}\right)+1 \mid$. Let $\left(A_{n}\right)_{n \in \mathbb{N}}$ and $\left(B_{n}\right)_{n \in \mathbb{N}}$ be given by (21) and (22), Then $\frac{A_{n}}{B_{n}}$ converge to some $\omega \in \mathscr{J}, D_{n}(\omega)=D_{n}$ and $h_{j}\left(D_{j}\right)=h_{j}\left(D_{j}(\omega)\right.$ for all $n \geq 1$.

Proof. Let $k \in \mathbb{N}$, we have

$$
\begin{aligned}
\left|\frac{A_{n+k}}{B_{n+k}}-\frac{A_{n}}{B_{n}}\right| & =\left|\sum_{i=n}^{n+k-1}\left(\frac{A_{i}}{B_{i}}-\frac{A_{i-1}}{B_{i-1}}\right)\right| \\
& \leq \max _{n \leq i \leq n+k-1}\left|\frac{A_{i}}{B_{i}}-\frac{A_{i-1}}{B_{i-1}}\right| \\
& <\max _{n \leq i \leq n+k-1} \frac{1}{\left|B_{i}\right|}=\frac{1}{\left|B_{n}\right|} \longrightarrow 0
\end{aligned}
$$

then $\frac{A_{n}}{B_{n}}$ is a cauchy sequence which implies that it converge. Let $\omega \in F_{q}\left(\left(X^{-1}\right)\right)$ be its limit.

Let us prove that $\omega \in \mathscr{J}, D_{n}(\omega)=D_{n}$ and $h_{n}\left(D_{n}(\omega)\right)=h_{n}\left(D_{n}\right)$. Since $\exists n_{0} \in N$ such that $\forall n \geq n_{0}$ we have $\left|\omega-\frac{A_{n}}{B_{n}}\right|<1$ then we obtain that

$$
|\omega| \leq \max \left(\left|\omega-\frac{A_{n}}{B_{n}}\right|,\left|\frac{A_{n}}{B_{n}}\right|\right)<1 .
$$

For the third part, let

$$
C_{n}=\left[0 ;\left(\begin{array}{c}
B_{1} \\
A_{1}
\end{array}\right), \ldots,\left(\begin{array}{c}
B_{n} \\
A_{n}
\end{array}\right)\right]=\frac{1}{B_{1}+A_{1}\left[0 ;\left(\begin{array}{c}
B_{2} \\
A_{2}
\end{array}\right), \ldots,\left(\begin{array}{c}
B_{n} \\
A_{n}
\end{array}\right)\right]}
$$

$=\frac{1}{B_{1}+A_{1} \widetilde{C}_{n}}$. It follows from the first part of the proof that there exists $\widetilde{\omega} \in \mathscr{J}$ such that $\lim _{n \rightarrow+\infty} \widetilde{C}_{n}=\widetilde{\omega}$. We find that $\omega=\frac{1}{B_{1}+A_{1} \widetilde{\omega}}$ which implies that $\widetilde{\omega}=\frac{\frac{1}{\omega}-B_{1}}{A_{1}} \in \mathscr{J}$. Since $D_{1}$ and $h_{1}\left(D_{1}\right)$ are unique for which $\frac{\frac{1}{\omega}-D_{1}}{h_{1}\left(D_{1}\right)} \in \mathscr{J}$, then $D_{1}=B_{1}$ et $A_{1}=h_{1}\left(D_{1}\right)$. By induction we find the result. 


\section{Competing interests}

The authors declare that they have no competing interests.

\section{Authors' contributions}

All authors have contributed to all parts of the article. All authors read and approved the final manuscript.

\section{References}

[1] Amara Chandoul, On periodic Jacobi-Perron algorithm over the field of formal power series. New Trends in Mathematical Science, v. 1, p. 145-152, 2018.

[2] Amara Chandoul, Hela Ben Amar and Mohamed Mkaouar, On the continued fraction expansion of fixed period in finite fields. (CMB) Canadian mathematical bulletin, 58(4):1-10, 2015. Doi: 10.4153/CMB-2015-055-9.

[3] Amara Chandoul, Hela Ben Amar and Mohamed Mkaouar, On periodic P-continued fraction having period length one. Bull. Korean Math. Soc. 50, No. 5, 1623-1630, 2013.

[4] Amara Chandoul, On continued fractions over the field of formal power series. Int. J. Contemp. Math. Sci. 6, No. 25-28, 1351-1356, 2011.

[5] Amara Chandoul, Simple proof of convergenvce of the Brun algorithm over the field of formal power series. (IJA) International Journal of Algebra, Vol. 5, no. 1, 25-30, 2011.

[6] Amara Chandoul and Hela Ben Amar, Convergenvce of the Brun algorithm over the field of formal power series. (JNT) Journal of Number Theory, 129, 621-631, 2009.

[7] L. Jacobsen, Convergence of limit k-periodic continued fractions $K\left(\frac{a_{n}}{b_{n}}\right)$, and of subsequences of their tails Proc. London Math. Soc. (3), 51 (1985), 563-576.

[8] Ai-Hua Fan, Bao-Wei Wang, Junn Wu, Arithmetic and metric properties of Oppenheim continued fraction, Journal of number theory 127 (2007), 64-82.

[9] W. J. Jones and W. J.Thron, Continued fractions, Analytic theory and applications, Encyclopedia of math 11 (Addison Wesley, Reading, Massachusetts, 1980).

[10] H. Waadeland, Tales about tails, Proc. Amer. Math. Soc, 90 (1984), 57-64.

[11] Amara Chandoul, Fractions continues multidimensionnelles: fractions continues multidimensionelles, polynômes irréductibles et nombres de Pisot. Editions universitaires europeennes, 2012, ISBN : 3838180542, 9783838180540. 